\title{
$\Gamma$ (2) MODULAR SYMMETRY, RENORMALIZATION GROUP FLOW AND THE QUANTUM HALL EFFECT
}

\author{
Yvon Georgelin $^{a}$, Thierry Masson ${ }^{b}$ and Jean-Christophe Wallet ${ }^{a}$
}

\author{
${ }^{a}$ Groupe de Physique Théorique, Institut de Physique Nucléaire \\ F-91406 ORSAY Cedex, France \\ ${ }^{b}$ Laboratoire de Physique Théorique (U.M.R. 8627), Université de Paris-Sud \\ Bât. 211, F-91405 ORSAY Cedex, France
}

\begin{abstract}
We construct a family of holomorphic $\beta$-functions whose $\mathrm{RG}$ flow preserves the $\Gamma(2)$ modular symmetry and reproduces the observed stability of the Hall plateaus. The semi-circle law relating the longitudinal and Hall conductivities that has been observed experimentally is obtained from the integration of the RG equations for any permitted transition which can be identified from the selection rules encoded in the flow diagram. The generic scale dependance of the conductivities is found to agree qualitatively with the present experimental data. The existence of a crossing point occuring in the crossover of the permitted transitions is discussed.
\end{abstract}

(May 1999)

LPT-99/02 


\section{INTRODUCTION}

The Quantum Hall Effect (QHE) is a remarquable phenomenon occuring in a twodimensional electron gas in a strong magnetic field at low temperature [1]. Since the discovery of the quantized integer [2] and fractional [3] Hall conductivity, the QHE has been an intensive field of theoretical and experimental investigations. The pioneering theoretical contributions [4] analyzing the basic features of the hierarchy of the Hall plateaus have triggered numerous works aiming to provide a better understanding of the underlying properties governing the complicated phase diagram associated with the quantum Hall regime together with the precise nature of the various observed transitions between plateaus and/or focusing on a characterization of a suitable theory.

It has been realized for some time that modular symmetries may well be of interest to understand more deeply salient properties of the QHE. For instance, the superuniversality proposed in [5] to explain the apparent similarity of the observed transitions is reminiscent to modular tranformations. Besides, it has been shown that some properties of the phase diagram may well be explained in terms of modular group transformations in a twoparameter scaling theory. At the present time, a fully satisfactory microscopic or effective theory for the QHE, from which the relevant modular symmetry (if any) would come out, is still lacking. This has somehow motivated studies focalized on the derivation of general constraints on the phase diagram (and/or expressions for the conductivities) coming from the full modular group [6] or some of its subgroups [7]. Indeed, it is well known that the existence of a discrete symmetry group acting on the parameter space of a theory induces restrictions on the renormalization group $(\mathrm{RG})$ flow. This has been pointed out $[6],[7]$ in the case of the full modular group which in that context can be viewed as a rich extension of the old Kramers-Wannier $Z_{2}$ duality of the two-dimensional Ising model. This interesting aspect has been applied in various areas of physics, such as statistical systems [8], extended Sine-Gordon theories [9], as well as non-perturbative analysis of $N=2$ supersymmetric Yang-Mills theory [10].

In this paper, we construct a familly of holomorphic $\beta$-functions which reproduces the observed stability of the Hall plateaus and whose corresponding RG flow in the conductivity plane (i.e. the parameter space) preserves a $\Gamma(2)$ symmetry acting on it. The paper is organized as follows. The section 2 is devoted to the construction. In section 3 , we discuss the corresponding physical consequences. We show in particular that the recently observed semi-circle law [11] relating the longitudinal and Hall conductivities can be recovered from the integration of the RG equations and that the predicted crossover for the various transitions is found to be in good qualitative agreement with the present experimental observations. We also compare our results to those obtained in a recent work dealing with the construction of a $\beta$-function based on another (larger) subgroup of the modular group [7c]. In section 4 , we collect the main results of this paper and we conclude. 


\section{CONSTRUCTION OF THE $\beta$-FUNCTIONS}

\subsection{Basic properties of $\Gamma(2)$}

The properties of the modular group $\Gamma(1)(\equiv P S L(2, Z))$ and its various subgroups can be found in [12]. In this section we collect all the relevant ingredients that we will use in the subsequent analysis. Let $\mathcal{P}$ and $z$ denote respectively the open upper-half complex plane and a complex coordinate on $\mathcal{P}(\operatorname{Im} z>0)$. One defines $\overline{\mathcal{P}} \equiv \mathcal{P} \cup Q$, where $Q$ is the set of rational numbers. We first recall that the group $\Gamma(2)$ is the set of transformations $G$ acting on $\overline{\mathcal{P}}$ defined by:

$$
\begin{gathered}
G(z)=\frac{a z+b}{c z+d}, \quad a, b, c, d \in Z, \quad(a, d) \text { odd and, }(b, c) \text { even } \\
a d-b c=1 \quad \text { (unimodularity condition) }
\end{gathered}
$$

$\Gamma(2)(\subset \Gamma(1))$, is the free group generated by

$$
T^{2}(z)=z+2, \quad \Sigma(z)=S T^{-2} S(z)=\frac{z}{2 z+1}
$$

where $T(z)=z+1$ and $S(z)=-\frac{1}{z}$ are the two generators of the modular group $\Gamma(1)$ and is known in the mathematical litterature as the principal congruence unimodular group at level 2. The corresponding principal fundamental domain $\mathcal{D}_{\Gamma(2)}$, depicted on fig.1, has three cusps denoted by $[0],[1] \simeq[-1]$ and $[i \infty]$ that are respectively identified with the three points 0,1 and $i \infty$ of $\overline{\mathcal{P}}$ which are the only fixed points of $\Gamma(2)$ on $\mathcal{D}_{\Gamma(2)}$. The whole set of fixed points of $\Gamma(2)$ on $\overline{\mathcal{P}}$ is obtained as usual by successive $\Gamma(2)$ transformations of these three pointsł. Notice the identification of the frontiers on $\mathcal{D}_{\Gamma(2)}$ as indicated on fig.1.

It has been pointed out recently in $[7 \mathrm{~b}, 13]$ that $\Gamma(2)$ can be used to derive a model for a classification of fractional (as well as integer) Hall states. This classification, which refines the Jain one [14] and involves a kind of generalization of the "law of the corresponding states" derived in [5], seems to reproduce successfully the observed hierarchical structure of the Hall states. The salient feature of the proposed construction is that each family of quantum fluid states indexed by fractions with odd denominators (plus the insulator state(s)) is generated from a metallic state labelled by an even denominator fraction through specific $\Gamma(2)$ transformations. To be more precise, we first rewrite the transformations $(2.1 \mathrm{a}, \mathrm{b})$ as

$$
G(z)=\frac{(2 s+1) z+2 n}{2 r z+(2 k+1)}, \quad(2 s+1)(2 k+1)-4 r n=1
$$

$\ddagger$ Observe that $\Gamma(2)$ has only real fixed points. 
where $k, n, r, s \in Z$. We identify for the moment $z$ with a filling factor $\nu=p / q$ and select a given Hall metallic state labelled by $\lambda=\frac{(2 s+1)}{2 r}(r \geq 0, s \geq 0)$. Then, as shown in [13], one obtains a hierarchy of Hall (liquid) states surrounding the metallic state $\lambda$ from the images $G_{n, k}^{\lambda}(0)$ and $G_{n, k}^{\lambda}(1)$ of 0 and 1 by the family of transformations $G_{n, k}^{\lambda} \in \Gamma(2)$ ( $n$ and $k$ satisfying (2.3b)) which sends $z=i \infty$ onto $\lambda$. As an exemple, the double Jain family of states surrounding the metallic state $\lambda=1 / 2$

$$
\begin{aligned}
& \frac{1}{3}, \frac{2}{5}, \frac{3}{7}, \ldots, \frac{N}{2 N+1} \\
& \frac{2}{3}, \frac{3}{5}, \frac{4}{7}, \ldots, \frac{N}{2 N-1}
\end{aligned}
$$

can be easily recovered in this scheme from the images $G_{n, k}^{1 / 2}(0)$ and $G_{n, k}^{1 / 2}(1)$ with respectively $n \geq 0$ for (2.4a) and $n<0$ for $(2.4 \mathrm{~b})$. We recall that this construction separates the even numerator Hall fractions from the odd numerator ones so that it may be possible to take into account a possible particle-hole symmetry within the present scheme. Other families surrounding any state indexed by an even denominator (metallic state) can be constructed in the same way so that all the experimentally observed Hall states can be taken into account in the present construction. It has been further shown in [7b] that the corresponding predicted global organization of the various Hall conductivity states stemming from the action of $\Gamma(2)$ fits quite well with (some of) the present experimental data.

The possible important role played by modular symmetries in the QHE has been considered for some time. Some of the related works have emphasized that (most of) the main features of the (up to now) experimentaly observed phase structure of QHE seem to be recovered from the action of suitable subgroup of the modular group on the complex conductivity plane hereafter identified with $\overline{\mathcal{P}}$ and parametrized by $z=\frac{\hbar}{e^{2}}\left(\sigma_{x y}+i \sigma_{x x}\right) \ddagger$ (with $\operatorname{Im} z \equiv \sigma_{x x} \geq 0$ ). In particular the group $\Gamma_{0}(2)$ has been mostly considered by some authors and used recently in [7c] to constraint the $\beta$-function governing the Renormalization Group (RG) flow of the conductivities for Quantum Hall systems. The corresponding studies have been performed under various physically acceptable set of hypothesis. In the next subsection, we will consider somehow similar hypothesis to study the restrictions which can be obtained from $\Gamma(2)$ on the $\beta$-function of the RG flow for the conductivities. We therefore assume that the action of $\Gamma(2)$ on real filling factors $\nu=p / q$ that has been described above can be extended to an action on $\overline{\mathcal{P}}$.

\subsection{Holomorphic $\beta$-functions from $\Gamma(2)$ symmetry}

$\ddagger$ in the following, $e^{2}=\hbar=1$ 
Let $t$ be a scale parameter whose possible explicit form will be specified in section 3. We first recall that scale transformations on the complex conductivity plane $\overline{\mathcal{P}}$ generate a $\mathrm{RG}$ flow $z \rightarrow R(t ; z, \bar{z}) \equiv z(t)$, from which the $\beta$-function is defined to be the (contravariant) vector field tangent to this flow, namely:

$$
\beta(z, \bar{z})=\frac{d R(t ; z, \bar{z})}{d t}=\frac{d z(t)}{d t}
$$

It is well known that the existence of a discrete symmetry group acting on the parameter space of a theory may induce restrictions on the RG flow and, in turn, provides some nonperturbative information on the RG flow (stemming basically from reasonable ansätze for the corresponding $\beta$-functions). As already mentionned in the introduction, this aspect has already been investigated in various areas of physics. Most of the considerations involved in these corresponding works can be adapted to the present situation for which we now outline the main steps of the analysis.

First of all, the crucial mathematical hypothesis is that the action of $\Gamma(2)$ commutes with the RG flow, which basically means that if the $\Gamma(2)$ symmetry of the parameter space (that is in the present case the conductivity plane) holds at a given scale, it will be preserved by the RG downwards to lower scales. This hypothesis in particular determines the $\Gamma(2)$-transformations of the $\beta$-function, given by

$$
\beta(G(z), \overline{G(z)})=(c z+d)^{-2} \beta(z, \bar{z})
$$

for any $G \in \Gamma(2)$, and may account for the apparent observed superuniversality in the Quantum Hall transitions [5]*.

Eqn. (2.6) indicates that $\beta$ transforms as a modular form of $\Gamma(2)$ with weight -2 whenever $\beta$ is holomorphic in $z$ on $\mathcal{P}$. In this later case, the applications of general results stemming from complex analysis permits one already to constraint strongly the possible expression for an admissible $\beta$-function. In the rest of this section, we will therefore assume that $\beta$ is holomorphic in $z$. This hypothesis will be commented upon in the beginning of section 3 .

Now, a general theorem on modular forms [12] states that any modular form $\omega(z)$ of any subgroup $\Gamma$ (of finite index) of the modular group with even weight $k$ can be represented on $\mathcal{D}_{\Gamma}$, the fundamental domain of $\Gamma$, as

$$
\omega(z)=\left(\lambda^{\prime}(z)\right)^{k / 2} R(\lambda)
$$

where $\lambda(z)$ is a modular function of $\Gamma \dagger$ defined on $\mathcal{D}_{\Gamma}, \lambda^{\prime}(z)=\frac{d \lambda(z)}{d z}$ and $R(\lambda)$ is a rational function in $\lambda$. In the present case, $k=-2$ and $\lambda$, the modular function of $\Gamma(2)$, can be

* It is easy to show that distinct critical points of the RG flow related by a $\Gamma(2)$ transformation will have the same scaling exponents.

$\dagger$ that is, a function invariant under the action of $\Gamma$ 
chosen as

$$
\lambda=\frac{\theta_{2}^{4}}{\theta_{3}^{4}}
$$

and satisfies on $\mathcal{D}_{\Gamma(2)}$

$$
\lambda(i \infty)=0, \lambda(0)=1, \lambda(1)=\infty
$$

In (2.8), the Jacobi $\theta$ functions $\theta_{2}$ and $\theta_{3}$ (together with $\theta_{4}$ given here for further convenience) are defined by

$$
\begin{gathered}
\theta_{2}=2 \sum_{n=0}^{\infty} q^{\left(n+\frac{1}{2}\right)^{2}}=2 q^{\frac{1}{4}} \prod_{n=1}^{\infty}\left(1-q^{2 n}\right)\left(1+q^{2 n}\right)^{2} \\
\theta_{3}=\sum_{n=-\infty}^{\infty} q^{n^{2}}=\prod_{n=1}^{\infty}\left(1-q^{2 n}\right)\left(1+q^{2 n-1}\right)^{2} \\
\theta_{4}=\sum_{n=-\infty}^{\infty}(-1)^{n} q^{n^{2}}=\prod_{n=1}^{\infty}\left(1-q^{2 n}\right)\left(1-q^{2 n-1}\right)^{2}
\end{gathered}
$$

where $q=\exp (i \pi z)$.

Therefore, consistency with the previous two hypothesis requires the general expression for the $\beta$-function on $\mathcal{D}_{\Gamma(2)}$ to be $\beta(z)=\lambda^{\prime}(z)^{-1} R(\lambda)$ with $\lambda$ given in (2.8), which can then be straightforwardly extended to $\mathcal{P}$ by the action of $\Gamma(2)$. We have now to confront this general expression to the experimental situation.

The strongest experimental constraint comes from the observed stability of the Hall plateaus labelled by integer as well as odd denominator fractional filling factors, which must presumably correspond to attractive stable fixed points of the $\beta$-function. To apply this constraint, we proceed as follows. First, observe that in the present framework, the fixed points of $\Gamma(2)$ must be by construction critical points of the $\beta$-function. On $\mathcal{D}_{\Gamma(2)}$, the only fixed points of $\Gamma(2)$ are 0,1 and $i \infty$ which can be respectively identified to the (Hall) insulator state, the first Landau level and some (unobserved) superconducting state. Next, observe that in the classification of the Hall states based on the $\Gamma(2)$ symmetry [7b,13], the Hall plateaus correspond to the images of 0 and 1 by $\Gamma(2)$ as recalled in section 2.1 (the images of $i \infty$ correspond to even denominator (metallic) Hall states). From these observations and under the further assumption that $\beta(z)$ has no other critical points than those given by the fixed points of $\Gamma(2)$, it is easy to realize that $\beta(z)$ can be conveniently parametrized on $\mathcal{D}_{\Gamma(2)}$ as

$$
\beta(z)=\frac{\alpha}{\lambda^{\prime}(z)} \lambda^{p}(z)(\lambda(z)-1)^{q}
$$

where $\alpha$ is a complex constant, $\lambda$ is still given by (2.8), $p, q \in Z$ and use has been made of (2.9). 
Now, according to the previous discussion, eqn.(2.11) must have zeros at $z=0$ and $z=1$. This is realized provided

$$
q \geq 1
$$

for $\beta(z=0)=0$ and

$$
p+q-1 \leq 0
$$

for $\beta(z=1)=0$. These constraints can be easily derived by combining (2.11) and (2.9) with the explicit expression for $\lambda^{\prime}(z)$ given by $\lambda^{\prime}(z)=i \pi \lambda(z) \theta_{4}^{4}(z)$, obtained from (2.8) and the functional relation

$$
\frac{\theta_{2}^{\prime}}{\theta_{2}}-\frac{\theta_{3}^{\prime}}{\theta_{3}}=\frac{i \pi}{4} \theta_{4}^{4}
$$

and making use of the following asymptotic expansions for the Jacobi $\theta$ functions:

$$
\begin{gathered}
\theta_{2}(z) \sim \sqrt{\frac{i}{z}}, \quad \theta_{3}(z) \sim \sqrt{\frac{i}{z}}, \quad \theta_{4}(z) \sim \sqrt{\frac{i}{z}} \exp \left(-\frac{i \pi}{4 z}\right) \quad \text { for } z \sim 0 \\
\theta_{2}(z) \sim \sqrt{\frac{i}{z-1}}, \quad \theta_{3}(z) \sim \sqrt{\frac{i}{z-1}} \exp \left(\frac{-i \pi}{4(z-1)}\right), \quad \theta_{4}(z) \sim \sqrt{\frac{i}{z-1}} \quad \text { for } z \sim 1
\end{gathered}
$$

¿From the combination of (2.11) with (2.12a) and (2.12b), one easily realize that $\beta(z)$ must be singular for $z=i \infty$, using for instance

$$
\theta_{2}(z) \sim \exp \left(\frac{i \pi z}{4}\right), \quad \theta_{3}(z) \sim 1, \quad \theta_{4}(z) \sim 1 \text { for } z \rightarrow i \infty
$$

a result that can be expected from general results on holomorphic functions. Finally, we find that the complex constant $\alpha$ appearing in (2.11) must be chosen to be real in order to obtain from the $\beta$-function a flow with the desired properties, in particular involving stable fixed points at values corresponding to the Hall plateaus. This is illustrated on fig. 2 and 3 where the flow of (2.11) with $q=1, p=0$ and $\alpha=-1$ is represented.

\section{DISCUSSION}

Let us first summarize what has been derived up to now. Putting (2.8), (2.11), (2.12) together with $\lambda^{\prime}(z)=i \pi \lambda(z) \theta_{4}^{4}(z)$, we finally obtain

$$
\begin{gathered}
\beta=i^{2 q-1} \frac{\alpha}{\pi} \frac{\theta_{2}^{4(p-1)} \theta_{4}^{4(q-1)}}{\theta_{3}^{4(p+q-1)}} \\
p, q \in Z, q \geq 1, p+q-1 \leq 0, \alpha<0
\end{gathered}
$$


which is defined on $\mathcal{D}_{\Gamma(2)}$ and can be straightforwardly extended to $\overline{\mathcal{P}}$. This is the main result of the previous section. Eqn (3.1) represents a physically admissible familly of holomorphic $\beta$-functions reproducing in particular the experimentally observed stability of the Hall plateaus and whose corresponding RG flow in the complex conductivity plane (i.e. the parameter space) preserves a $\Gamma(2)$ symmetry acting on it.

In this section we will show that the physical predictions that can be extracted from (3.1) are in good agreement with the present experimental observations. Namely, we will show that the "semi-circle"law which has been recently observed [15] can be recovered from the behaviour of $\sigma_{x y}$ and $\sigma_{x x}$ obtained from the integration of (3.1). We will also show that the crossover in the plateau-plateau and plateau-insulator (observed) transitions described by (3.1) agrees qualitatively with the present experimental observations.

Before starting the discussion, some important remarks concerning the holomorphy hypothesis as well as the already proposed candidates for $\beta$-functions are in order. As it is well known, the holomorphy constraint is a very strong one which restricts severely the possible expression for $\beta$. Relaxing this constraint permits one to have much more freedom for the construction of physically admissible $\beta$-functions. For a recent comprehensive analysis of the non-holomorphic case, see e.g. [16] and references therein. Here, we notice that non-holomorphic $\beta$ that have been proposed in [16] can obviously be related through their asympotic (large $\sigma_{x x}$ ) behaviour to the $\beta$-functions stemming from the dilute-instanton gas calculations performed in the framework of non-linear sigma-models [17], whereas the family of holomorphic $\beta$-functions given in (3.1) cannot be. In particular, the corresponding asymptotic (large $\sigma_{x x}$ ) behaviour is different and indeed cannot be finite as it is for those non-holomorphic $\beta$ 's. Imposing finitude at large $\sigma_{x x}$ for (3.1) would produce necessarely unstable Hall plateaus, in clear contradiction with the experimental observations. Therefore, one of the characteristic features of (3.1) is the existence of a singularity as $\sigma_{x x} \rightarrow \infty$ and consequently at any even denominator rational point on the real axis in the conductivity plane. Anticipating the discussion, the physical consequence on the corresponding flow diagram is the existence of unstable path connecting even denominator (metallic) Hall states to odd denominator (or insulator) states which might be associated to "unfavored" (but nevertheless observable) transitions.

Let us now turn to already proposed candidates for a $\beta$-function and RG flows. Recall that the early developments on this subject were essentially based on a field theory of the type of non linear sigma model [17] (see also [18]) mentionned just above with two (dimensionless) coupling constants identified with the longitudinal $\left(\sigma_{x x}\right)$ and Hall $\left(\sigma_{x y}\right)$ conductivities of the disorderd electron gas. Starting from this framework, a RG flow diagram for the conductivities has been conjectured [18] whose characteristic feature is the existence of fixed points occuring at some $\sigma_{x x}$ and $\sigma_{x y}$ equal to half integer values. Althought this proposal is appealing and seems to capture some experimental features of the (mainly integer) Quantum Hall Effect, it is plagued with a problem. Indeed, the 
postulated fixed points (if they exist as fixed points of the non linear sigma model [17], a fact which is not clear at the present time) correspond to a small $\sigma_{x x}$ (strong coupling) regime whereas the dilute-instanton gas calculation that gives rise to the non-holomorphic $\beta$-function underlying the conjectured RG flow is valid only in the large $\sigma_{x x}$ (weak coupling) regime. Whether this crude extrapolation from the weak to the strong coupling regime is finally correct or not is unclear at the present time. Keeping in mind the above remarks and that there is no experimental facts favoring either holomorphic or non-holomorphic $\beta$ as far as we know, one can reasonably regard the family of holomorphic $\beta$ 's (3.1) based on $\Gamma(2)$ as possible candidates for a description of aspects of the physics of the QHE effect.

We close these remarks by noticing that similar constructions of holomorphic $\beta$ functions based on larger subgroups of the full modular group have been performed recently, most of them focussing on the subgroup $\Gamma_{0}(2)$. Recall that $\Gamma_{0}(2)$ is generated by $T(z)=z+1$ and $\Sigma(z)=z /(2 z+1)$ and that one has $\Gamma(2) \subset \Gamma_{0}(2)$, i.e. $\Gamma(2)$ is a subgroup of $\Gamma_{0}(2)$. It has been shown $[7 \mathrm{c}]$ that a $\Gamma_{0}(2)$-based construction gives rise to a qualitative behaviour of the crossover in the various transitions that is similar to the one obtained from our $\Gamma(2)$-based construction. There are however specific differences appearing in the corresponding flows. In particular, one of the fixed point of $\Gamma_{0}(2)$ in its fundamental domain has to be identified naturally with the crossing point appearing in the plateau-insulator transitions, whose position is therefore entirely ("rigidely") determined in the conductivity plane. Recall that it corresponds to $\sigma_{x y}=1 / 2$ and $\sigma_{x x}=1 / 2\left(z=\frac{1+i}{2}\right)$ for the $0 \rightarrow 1$ transition. Moreover, the proposed $\beta$-function has a pole at this point. In that case, the unique path in the conductivity plane connecting $z=0$ to $z=1$, which must go obviously through the pole $z=\frac{1+i}{2}$, appears to be unstable (and indeed can evolve either from 0 toward $\infty$ or to 0 toward $1 / 2$ ), a fact that can be easily verified numerically by plotting the flow generated by the corresponding $\beta$. Further investigations are needed to clarify the experimental and theoretical status of this predicted crossing point. In the $\Gamma(2)$ case, the situation is quite different. Indeed, the point $z=\frac{1+i}{2}$ does not play a distinguished role simply because it is not a fixed point of $\Gamma(2)$ so that consequently it can be neither a zero nor a pole of the corresponding $\beta$ in the present framework.

Let us now examine critically the physical consequences encoded in (3.1). First, notice that $\beta$-functions defined by (3.1) and such that $p+2 q-2=0$ holds generate a flow approaching $z=0$ and $z=1$ in the same manner. This can be easily seen by combining (3.1) with $(2.14 \mathrm{a}, \mathrm{b})$ and studying the behaviour of $\beta$ in the vicinity of $z=0$ and $z=1$. In particular, it can be straightforwardly realized that the fastest approach of 0 and 1 is obtained when $q=1$ and $p=0$ which corresponds to asymptotic expressions for $\beta$ that do not involve exponential factors. From now on we will focuss on this later situation.

Now, we point out that the RG equation can be formally integrated along the single trajectories in the parameter space, leading to an algebraic relation between $\sigma_{x y}$ and $\sigma_{x x}$ which reproduces the "semi-circle" law that has been experimentaly observed, at least at 
low temperature, in the study of the plateau-insulator and plateau-plateau transitions [15]. Indeed, combining (2.5) with (2.11) (in which now $p=0, q=1$ and we take $\alpha=-1 \dagger$ ), one obtains

$$
d t=-\frac{d \lambda}{\lambda-1}
$$

where $\lambda$ is still given by (2.8). The integration of (3.2) gives

$$
t=-\log (\lambda-1)+\chi
$$

where $\chi$ is a complex constant and log denotes a determination of the complex logarithm. It follows, for any determination of the logarithm, that

$$
\lambda=1+\exp (\chi-t)
$$

and one has $\lambda \rightarrow 1$ (resp. $+\infty)$ for $t \rightarrow+\infty$ (resp. $-\infty$ ).

Let us assume $\chi$ real so that $\lambda$ is real for any $t$. Then, it is a general result that the inversion of the map $z(t) \mapsto \lambda(z(t))=1+\exp (\chi-t)$, with $\lambda$ given by (2.8), gives rise to a curve $t \mapsto z(t)$ which is a semi circle linking two rational numbers as end-points on the real axis together with a point $z_{0}$ located on this semi-circle and satisfying $\lambda\left(z_{0}\right)=1+\exp \chi$. The inversion of this map does not result in a unique semi-circle because if $t \mapsto z(t)$ is a solution then, for any $G \in \Gamma(2), t \mapsto G(z(t))$ is also a solution (whose end-points on the real axis are therefore the images by $G \in \Gamma(2)$ of the end-points of the initial solution). But it can be easily realized that the choice of such a solution among all the possible one is completely equivalent to the choice of a $z_{0}$ such that $\lambda\left(z_{0}\right)=1+\exp \chi$, which can be regarded as initial conditions for the $R G$ equation.

It is instructive to attempt to make a closer contact with the experimental situation throught the following (more physical) interpretation for $z_{0}$. Observe first that one should have $t=0$ at $z=z_{0}$. Assume then that $t$ can be cast into the form [19]

$$
t=f\left(\frac{1}{T^{\gamma}}\left(\frac{1}{B}-\frac{1}{B_{c}}\right)^{\delta}\right)
$$

at least in the domain of interest, a form which is inspirated from a two-parameter scaling framework, where $f$ is a monotonic function with $f(0)=0, B_{c}$ is a critical value for the external magnetic field $B$ and $T$ is the temperature. This therefore suggests that $z_{0}$ would correspond to the point on the semi-circle where $B=B_{c}$, independantly of the temperature, so that $z_{0}$ might be naturally identified with a crossing point appearing in the crossover between the two Hall states whose filling factors correspond to the rational points on the real axis connected by the semi-circle.

\footnotetext{
$\dagger$ other real value for $\alpha$ corresponds to simple rescaling of $t$ so that the ensuing analyzis is not modified
} 
To illustrate the previous discussion, a representative exemple of the resulting flow is depicted on figure 2 where only semi-circle line flows are considered. The plateau-insulator transition $0 \rightarrow 1$ corresponds to the (full line) large semi-circle linking 0 and 1 . As it should be clear now, the action of successive $\Gamma(2)$ transformations on this semi-circle (which may be viewed as a template for the transitions) gives rise to the other (full-line) semi-circles appearing on this figure. For instance, the following transformation $G(z)=\frac{3 z-2}{2 z-1} \in \Gamma(2)$ maps the semi-circle for the $0 \rightarrow 1$ transition into the semi-circle connecting 1 and 2 , which therefore corresponds to a direct transition between the Hall plateaus with integer filling factor $\nu=1$ and $\nu=2$, whereas $G(z)=\frac{z}{2 z+1} \in \Gamma(2)$ maps the $0 \rightarrow 1$ semi-circle into the one associated to the transitions $0 \rightarrow 1 / 3$. Therefore, if the present framework is correct, it is expected that such a semi-circle law should be experimentally observed for any other permitted transitions. This is already the case for the observed $0 \rightarrow 1 / 3,0 \rightarrow 1$ and $1 \rightarrow 2$ transitions that have been recently studied in e.g. some Si MOSFET devices in the Quantum Hall regime [20,15b] and for which the data on $\sigma_{x x}$ and $\sigma_{x y}$ fit well with the semi-circle law expected from the present $\Gamma(2)$-based framework. Notice by the way that selection rules for the permitted direct plateau-plateau and plateau-insulator transitions can be obviously extracted from the flow diagram depicted on fig.2 by simply observing that each permitted transition is rigidely linked with a semi-circle whose end-points on the real axis correspond to the filling factors labelling the transition.

The above analysis can be easily adapted to the case where $\chi$ is a complex number. In this later situation, the trajectories $t \mapsto z(t)$ (each of which still connecting two rational numbers on the real axis) are no longer semi-circles, as depicted on fig.3.

As announced in the beginning of this section, there are a priori possible transitions of the type insulator $\rightarrow$ even denominator (metallic) state as well as odd denominator $\rightarrow$ metallic state, namely $0 \rightarrow 1 / 2,1 \rightarrow 1 / 2$ and the corresponding images by $\Gamma(2)$. Some representative examples of these transitions are indicated on fig. 2 and 3 by dashed lines $\dagger$. The associated trajectories are in fact unstable as any small deviation from the semi-circle connecting, says 0 to $1 / 2$ will give rise to a quite different transition. For instance, it can be easily realised from fig. 3 that a small perturbation of the flow line associated to the $0 \rightarrow 1 / 2$ transition will give rise to either the $0 \rightarrow 1$ or $0 \rightarrow 1 / 3$ transition, whereas any plateau-plateau as well as plateau-insulator transition is stable against perturbation.

The explicit expressions for $\sigma_{x x}$ and $\sigma_{x y}$ as functions of $t$, which are expected to provide the qualitative behaviour of the crossover for a transition, can be easily extracted from (3.2)-(3.4). The method is standard and is essentially similar to the one used in [7c]. The resulting expressions are similar to the one given in [7c]. It is however interesting to plot

$\dagger$ Notice that there exists also, as a consequence of the present construction, transitions of the type $0 \rightarrow \infty$ or $1 \rightarrow \infty$ and the corresponding $\Gamma(2)$ images. The state $\sigma_{x x}=\infty$ would represent some (yet unobserved) supraconducting state. 
them numerically. This is done on fig.4 (resp. fig.6) for the $1 \rightarrow 0$ (resp. $1 \rightarrow 2$ ) transition, whereas fig. 5 and fig. 7 represent the $t$-dependance of the corresponding resistivities. It can be realized that the predicted behaviour of the conductivities (as well as the resistivities) is in good qualitative agreement with the experimental one reported in particular in [15a,b]. We observe by the way that the "almost linear" asymptotic shape of $\rho_{x x}(t)$ for $t>0$ that we obtain fits well with a recent experimental measurement of the corresponding quantity for the $1 \rightarrow 0$ transition [15b] (keeping in mind the possible interpretation for $t$ parametrized by (3.5)). Notice that somehow similar results for the crossovers have been obtained in $[7 \mathrm{c}]$ where the symmetry $\Gamma_{0}(2)$ is considered instead of $\Gamma(2)$.

As a last remark, notice that a precise experimental determination of the location of the crossing point on the semi-circular trajectories in the conductivity plane would be helpful for the identification of the possibly relevant modular subgroup. Indeed, a crossing point found to be really located on the uppermost point of the semi-circle (e.g. corresponding to $z=\frac{1+i}{2}$ for the $0 \rightarrow 1$ transition) would favor the group $\Gamma_{0}(2)$. On the other hand, a crossing point found to deviate significantly from the uppermost location would favor $\Gamma(2)$ (putting therefore $\Gamma_{0}(2)$ into trouble). This later case seems to have been recently observed in the experiment performed in the second ref.[15b]. Note that, in this experiment, the corresponding candidates for the crossing points in the $0 \rightarrow 1$ and $0 \rightarrow 1 / 3$ transitions can be related to each other (within, says, $\sim 10 \%$ ) by a $\Gamma(2)$ transformation, as it must be the case in the present framework.

\section{CONCLUSION}

Let us summarize in physical words the main results of this paper. We have proposed a (familly of) holomorphic $\beta$-function(s) whose RG flow preserves the $\Gamma(2)$ modular symmetry and which is consistant with the observed stability of the Hall plateaus. The semi-circle law relating the longitudinal and Hall conductivities that has been observed experimentally for the $0 \rightarrow 1,0 \rightarrow 1 / 3$ and $1 \rightarrow 2$ transitions is obtained from the integration of the $\mathrm{RG}$ equations for these transitions and, in fact, must hold in the present framework for any permitted transition which can be easily identified from the selection rules encoded in the flow diagram. Moreover, it has been shown that there exists a unique point on each semi-circle where the generic scale parameter $t$ vanishes. This combined with a two-parameter scaling hypothesis, as an additional phenomenological input (yielding a plausible parametrization for $t$ given by (3.5)), suggests to interpret this point as the crossing point occuring in the crossover of the two Hall states involved in the transition. The generic scale dependance of the conductivities has been verified to agree qualitatively with the present experimental data. In the present framework, the trajectories in the conductivity plane involving 
an even denominator filling factor are found to be unstable. Although we do not have a clear interpretation (if any) of this, one might expect that the corresponding (observed) transitions are unfavored. 


\section{REFERENCES}

1) The Quantum Hall Effect, 2nd ed., R.E. Prange and S.M. Girvin eds. (SpringerVerlag, New-York) 1990. See also Perspectives in Quantum Hall Effect, S.D. Sarma and A. Pinczuk eds. (Wiley, New York) 1997.

2) K.von Klitzing, G. Dorda and M. Pepper, Phys. Rev. Lett. 45 (1980) 494.

3) D.C. Tsui, H.L. Störmer and A.C. Gossard, Phys. Rev. Lett. 48 (1982) 1559.

4) R.B. Laughlin, Phys. Rev. Lett. 50 (1983) 1385; F.D. Haldane, Phys. Rev. Lett. 51 (1983) 605; B.I. Halperin, Phys. Rev. Lett. 52 (1984) 1583.

5) S. Kivelson, D.H. Lee and S.C. Zhang, Phys. Rev. B46 (1992) 2223.

6) C.A. Lütken and G.G. Ross, Phys.Rev. B45 (1992) 11837, Phys. Rev. B48 (1993) 2500; see also E. Fradkin and S. Kivelson, Nucl. Phys. B474 (1996) 543.

7a) C.A. Lütken, Nucl. Phys. B396 (1993) 670.

7b) Y. Georgelin, T. Masson and J.C. Wallet, J. Phys. A: Math. Gen. 30 (1997) 5065.

7c) B.P. Dolan, Modular invariance, Universality and crossover in the QHE, cond-mat/9809294: B.P. Dolan, Duality and the modular group in QHE, cond-mat/9805171.

8) J. Cardy, Nucl. Phys. B203 (1982) 17; J. Cardy and E. Rabinovici, Nucl. Phys. B203 (1982) 1.

9) D. Carpentier, J. Phys. A: Math.Gen.32 (1999) 3865 and references therein.

10) N. Seiberg and E. Witten, Nucl. Phys. B426 (1994) 19; J.I. Latorre and C.A. Lütken, Phys. Lett. B421 (1998) 217; A. Ritz, Phys. Lett. B434 (1998) 54.

11) A.M. Dykhne and I.M. Ruzin, Phys. Rev. B50 (1994) 2369; I.M. Ruzin and S. Feng, Phys. Rev. Lett. 74 (1995) 154.

12) R.A. Rankin, Modular Forms and Functions, Cambridge University Press, 1977.

13) Y. Georgelin and J.C. Wallet, Phys. Lett. A224 (1997) 303.

14) J.K. Jain, Phys. Rev. B41 (1990) 7653.

15a) M. Hilke et al., Nature 395 (1998) 675.

15b) M. Hilke et al., Semi circle: An exact relation in the Integer or Fractional Quantum Hall Effect, cond-mat/9810217.

16) C.P. Burgess and C.A. Lütken, On the implication of discrete symmetries for the $\beta$-function of Quantum Hall System, cond-mat/9812396.

17) A.M.M. Pruisken quoted in ref. [1a], Phys. Rev. B32 (1985) 2636, Nucl. Phys. B285 (1987) 61.

18) D.E. Khmel'niskii, Pisma Zh. Eksp. Teor. Fiz.38 (1983) 454.

19) For a general discussion see B. Huckestein, Rev. Mod. Phys. 67 (1995) 357.

20) D. Shahar et al., Phys. Rev. Lett. 79 (1997) 479. 


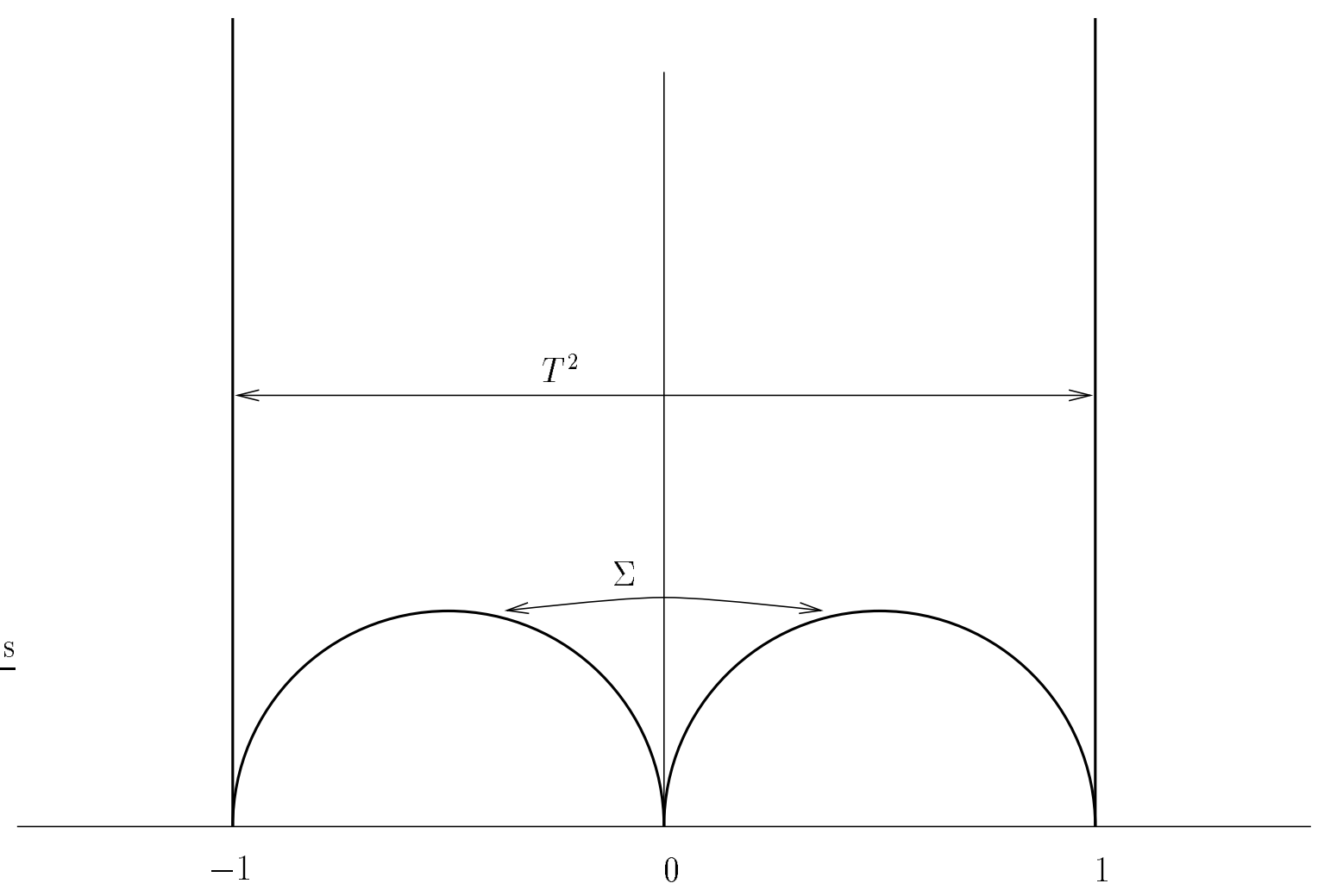

Figure 1: The principal fundamental domain of $\Gamma(2)$. The arrows indicate the frontiers which has to be identified.

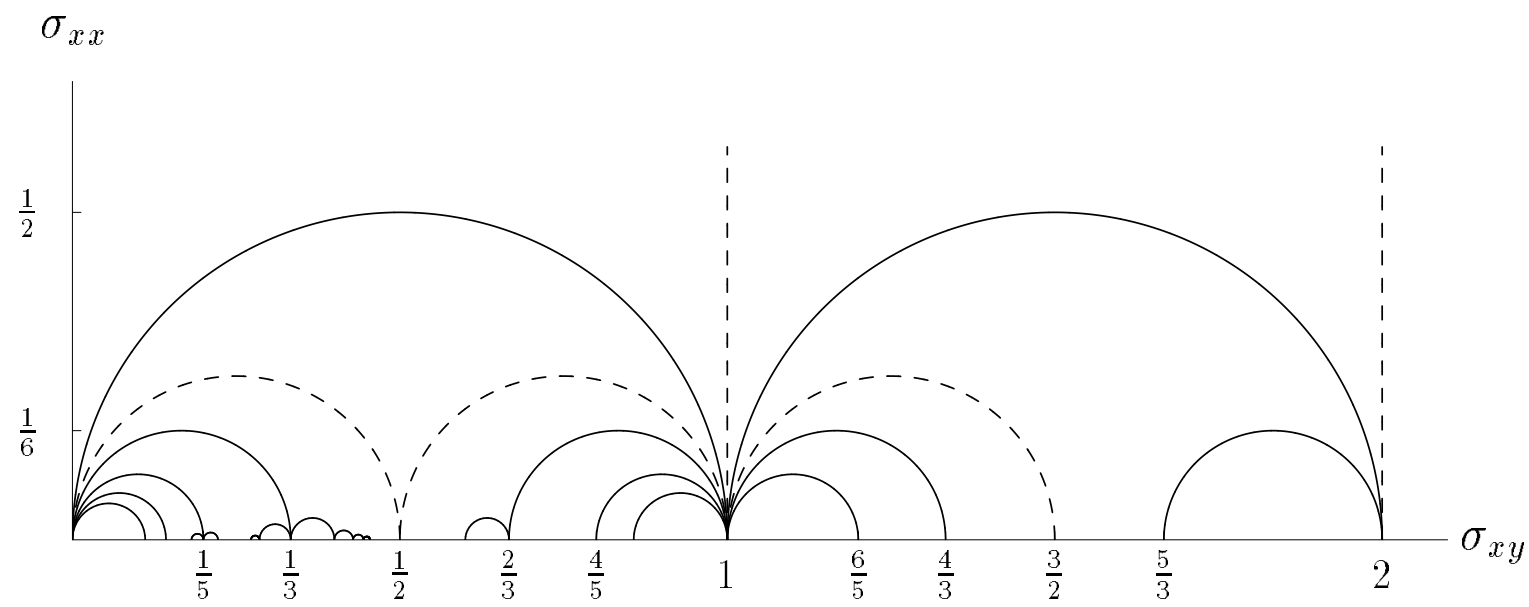

Figure 2: Some semi-circle lines flow for the $\beta$ function corresponding to $\alpha=-1, p=0$ and $q=1$. Solid lines correspond to plateau-plateau or plateau-insulator transitions. Dashed lines indicate unstable transitions. 


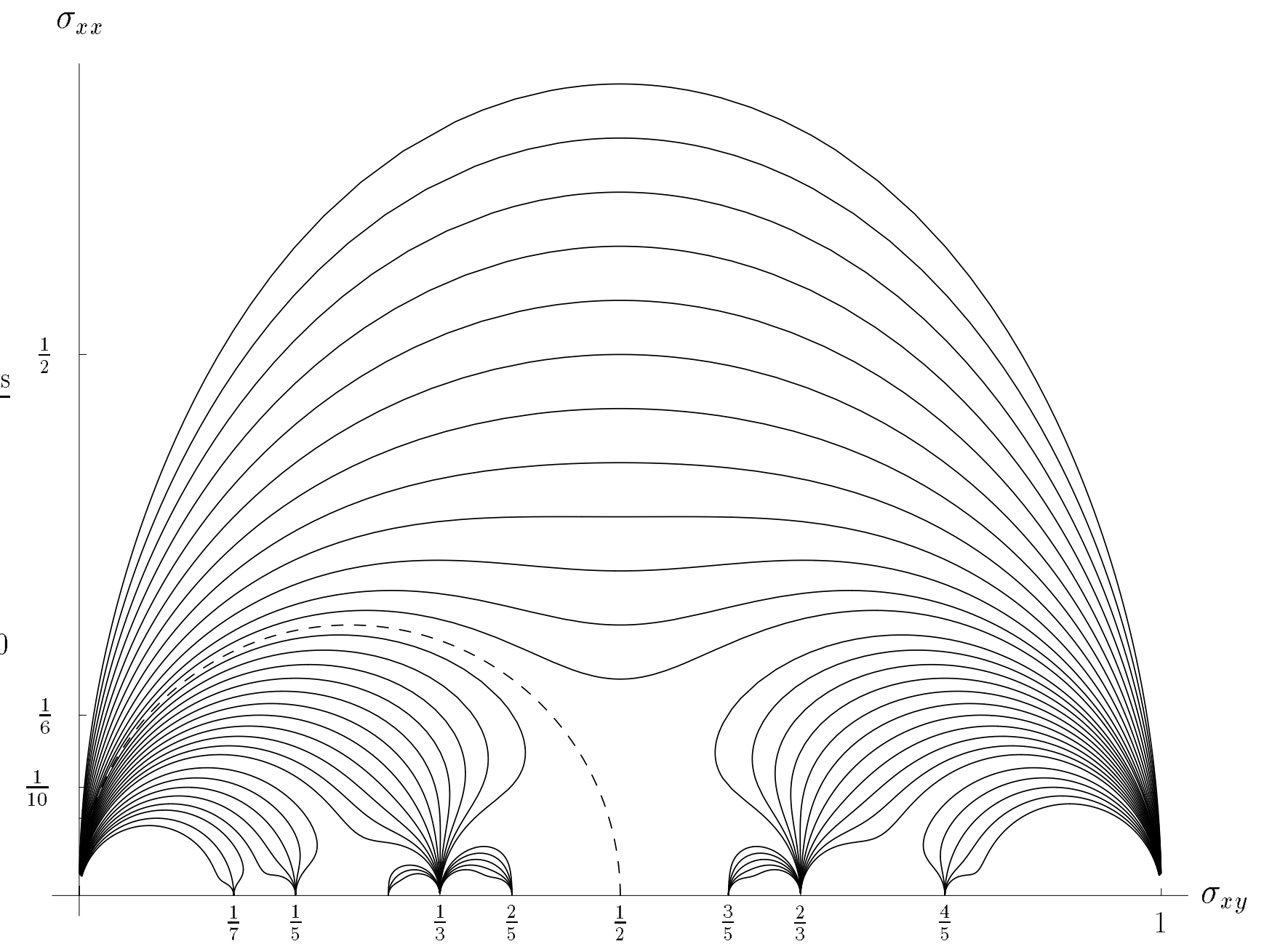

Figure 3: Some general lines flow for the $\beta$ function corresponding to $\alpha=-1, p=0$ and $q=1$. Solid lines correspond to plateau-plateau or plateau-insulator transitions. Dashed lines indicate unstable transitions. 

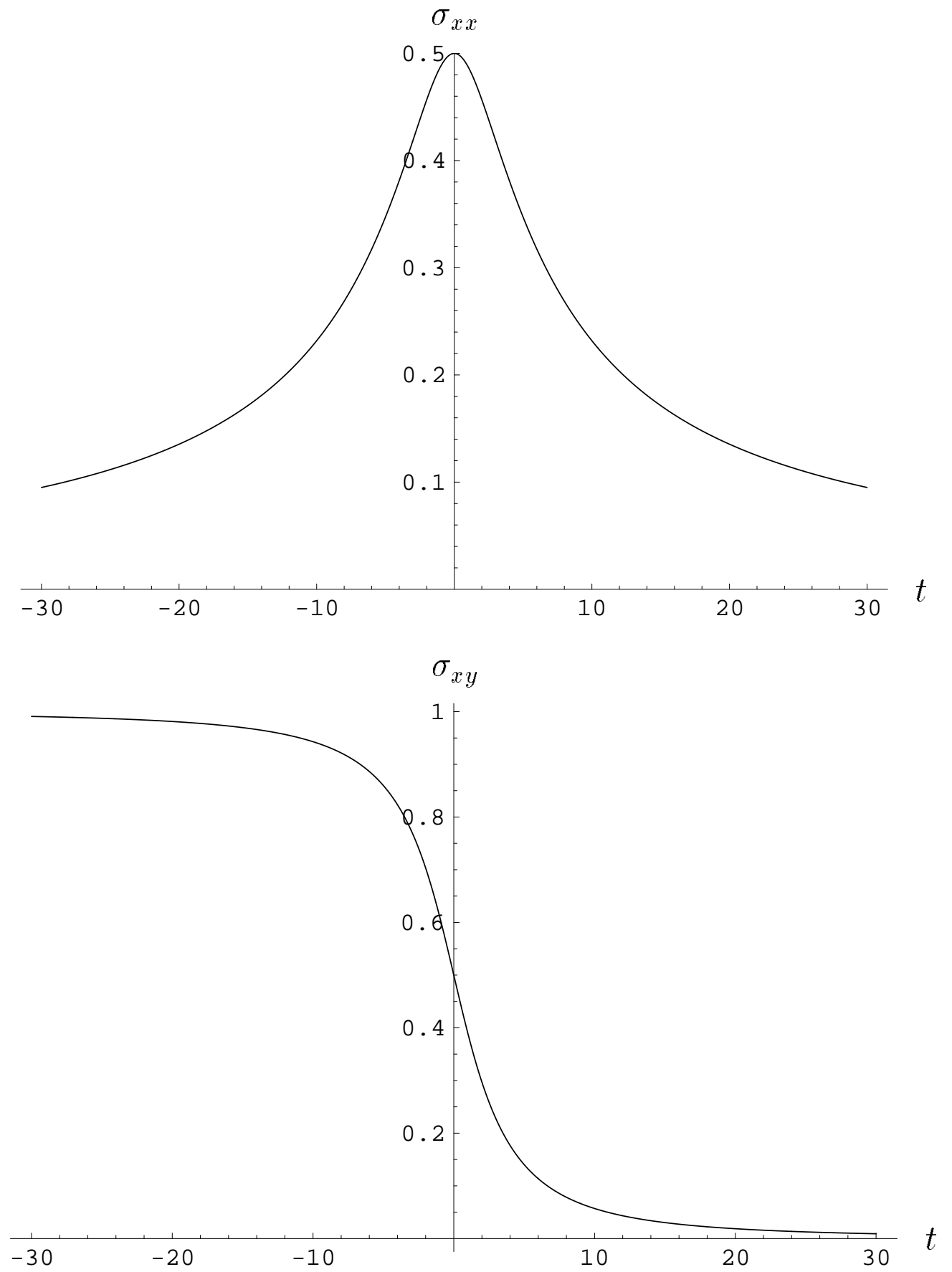

Figure 4: $\sigma_{x x}$ and $\sigma_{x y}$ versus scale parameter $t$ for the transition $1 \rightarrow 0$. 


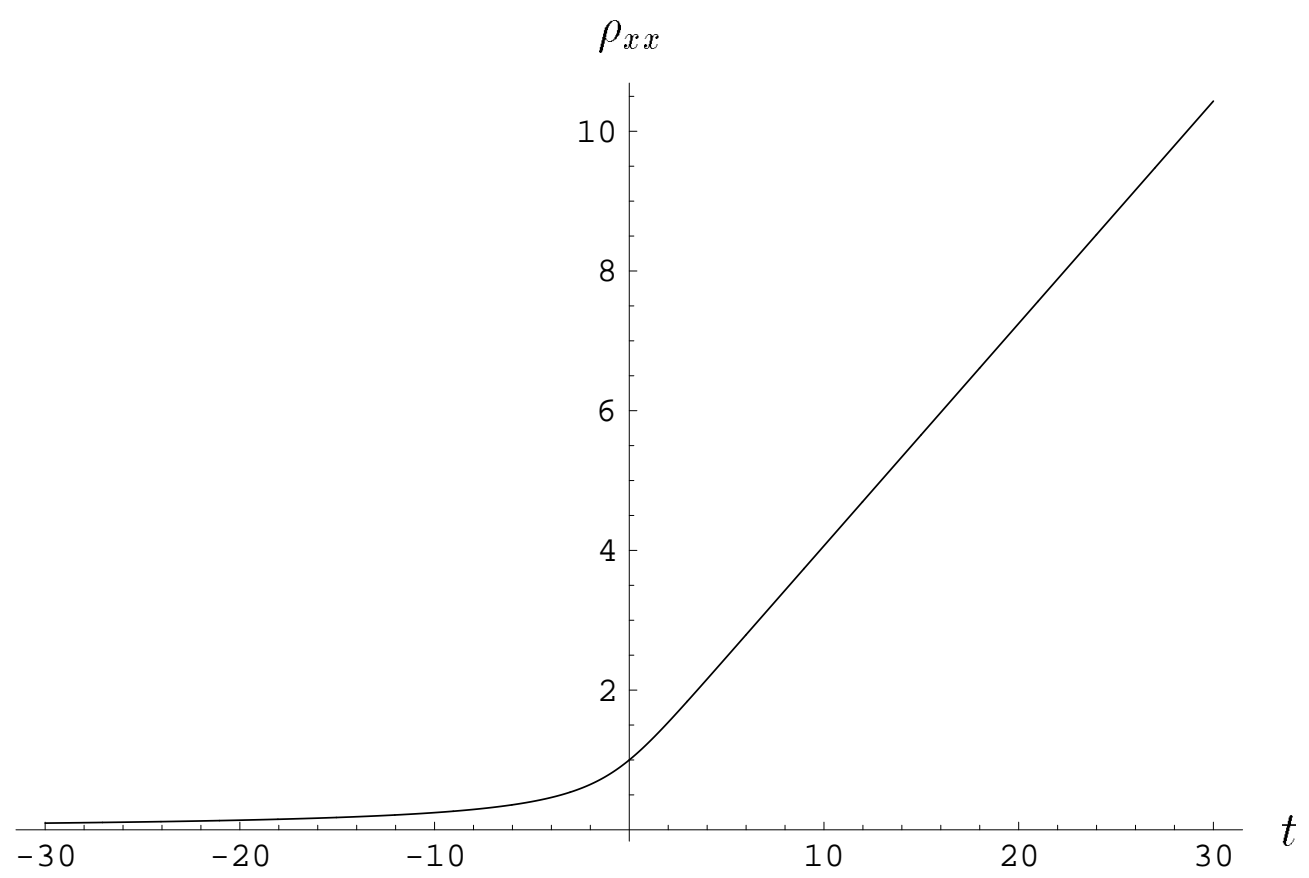

Figure 5: $\rho_{x x}$ versus scale parameter $t$ for the transition $1 \rightarrow 0$. 


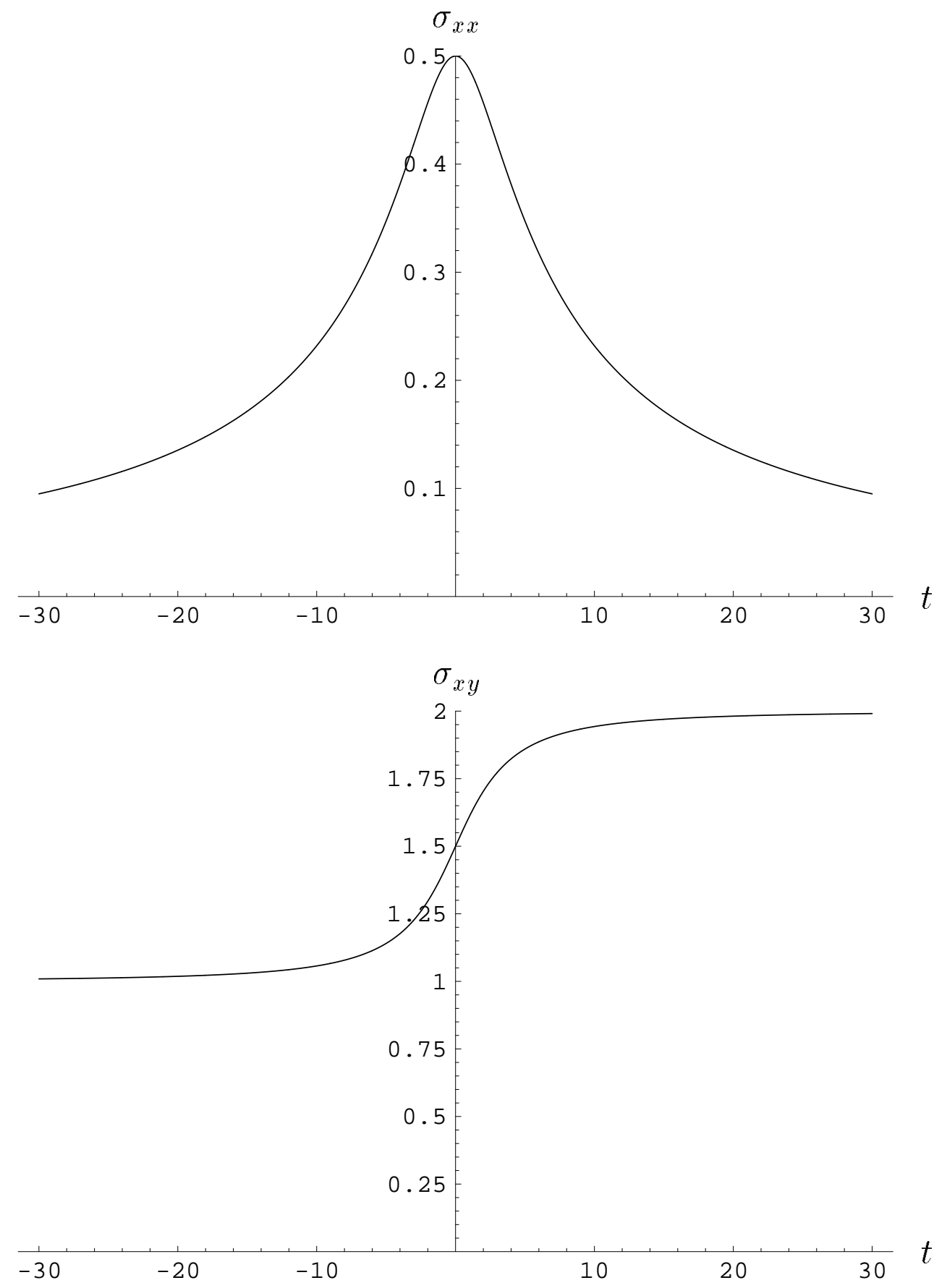

Figure $6: \sigma_{x x}$ and $\sigma_{x y}$ versus scale parameter $t$ for the transition $1 \rightarrow 2$. 


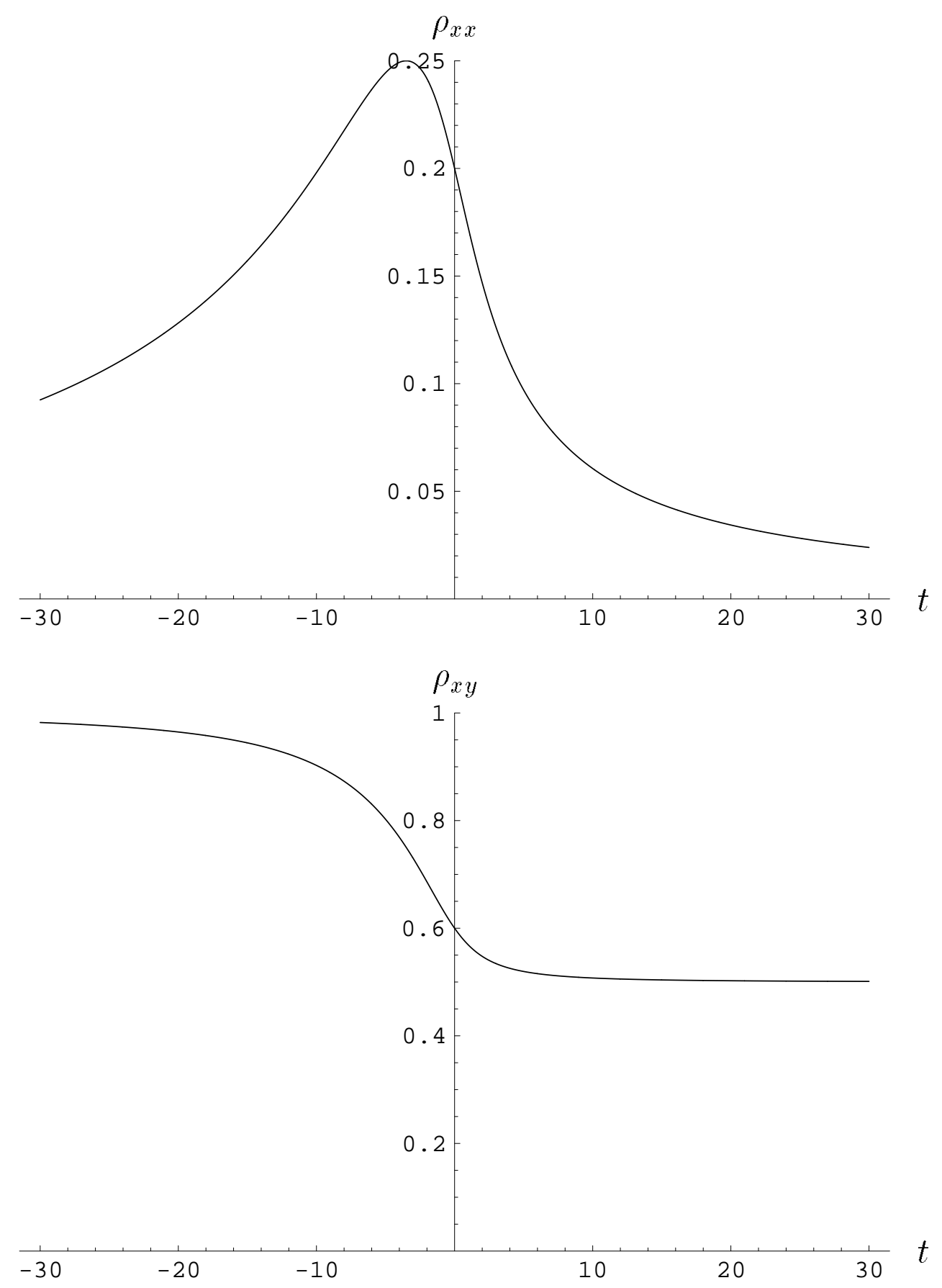

Figure 7: $\rho_{x x}$ and $\rho_{x y}$ versus scale parameter $t$ for the transition $1 \rightarrow 2$. 